\title{
STUDIES ON ICTERUS NEONATORUM
}

\section{The Production of icterus in Antmals Following Prolonged ANOXAEMTA $^{1}$}

By ALTON GOLDBLOOM AND RUDOLF GOTTLIEB

(From the Department of Medicine, McGill University Clinic, Royal Victoria Hospital, Montreal)

(Received for publication October 9, 1929)

In a previous paper (1) in which we reported our studies on icterus neonatorum, we demonstrated the readiness with which the blood of a newly born infant haemolysed as compared with the blood of an older child or an adult. We showed also that the morphologic pattern of the blood of the new born was considerably different; for instance the presence of a polycythaemia and the increase in the number of nucleated red cells and reticulocytes. We showed too, that reduction of the polycythaemia and of the number of immature cells in the circulation coincided with an increase in the bilirubin in the serum, the development of icterus and the development of a normal resistance of the red cells in varying concentrations of salt solution. We showed also that the diminished resistance of the red cells of the newly born to salt solution is due to the immature cells in the circulation, namely that these cells haemolyse even in normal salt solution, and that the cells remaining after these immature forms have been destroyed, show normal resistance.

In attempting to explain the nature of icterus neonatorum, we reasoned that, in view of the admixture of arterial and venous blood, the right to left shunt existing in ante natal life, the foetus, was comparable to any other individual living in a state of diminished oxygen supply. That immediately after birth, with the cessation of admixture in normal infants, the necessity for polycythaemia no longer

1 This research was made possible by a grant from the Cooper Fund. 


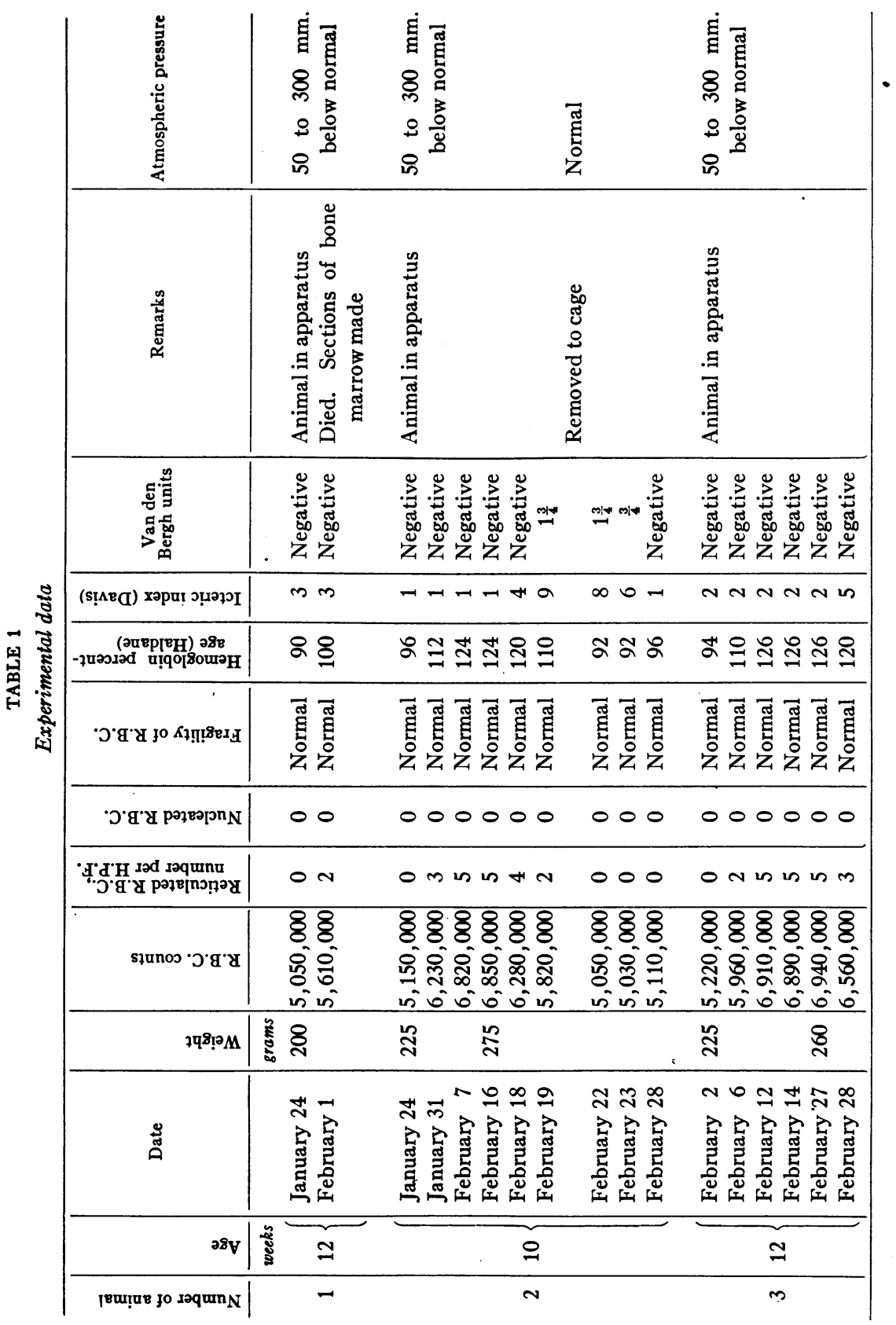




\begin{tabular}{|c|c|c|c|c|c|c|}
\hline 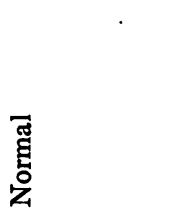 & 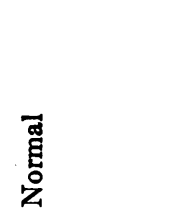 & & 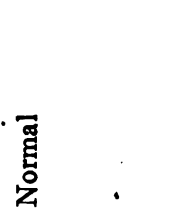 & & 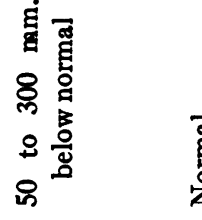 & 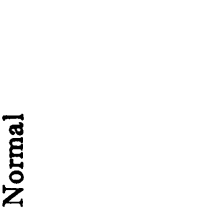 \\
\hline 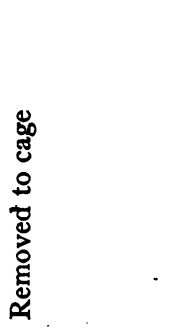 & 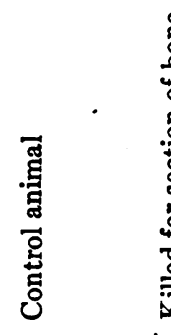 & 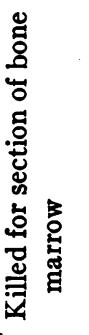 & 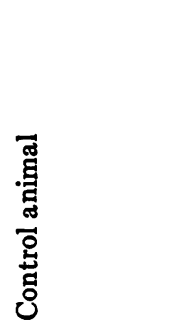 & 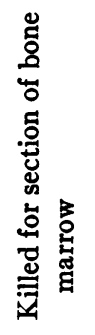 & 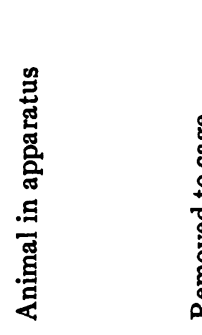 & 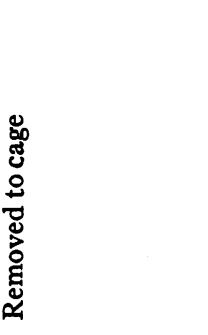 \\
\hline \#- & 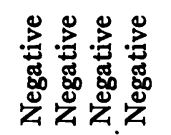 & & 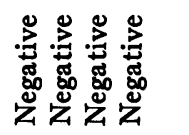 & & 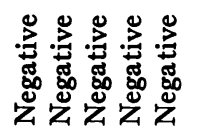 & 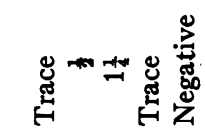 \\
\hline$\infty \backsim N N$ & 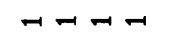 & & NNNN & & 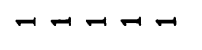 & n $00 \mathrm{mr}$ \\
\hline 요 & สูสสู & & สูะสสส & & 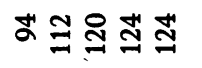 & 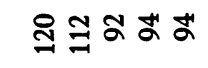 \\
\hline 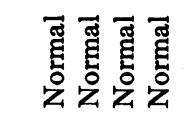 & 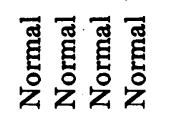 & & 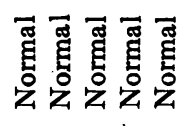 & & 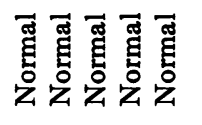 & 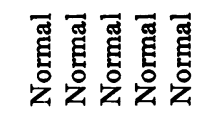 \\
\hline 0000 & 0000 & & 00000 & & 00000 & 00000 \\
\hline NoOO & 0000 & & 00000 & & Omt in in & introo 0 \\
\hline 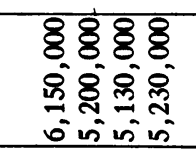 & 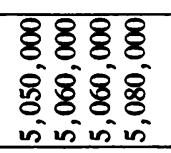 & & 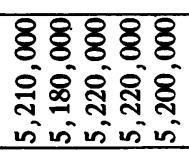 & & 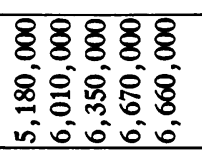 & 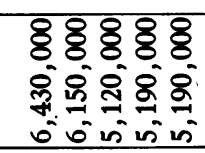 \\
\hline & ళ్ల \&్ন & & ஓ \& & & 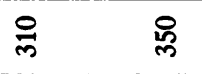 & \\
\hline 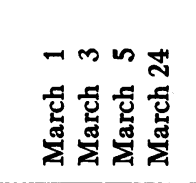 & 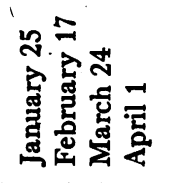 & & 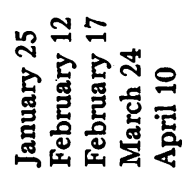 & & 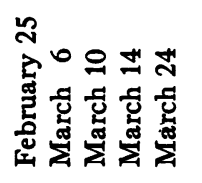 & 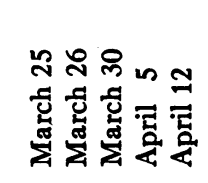 \\
\hline$\approx$ & $=$ & 、 & \pm & & & \pm \\
\hline $\bar{m}$ & - & & in & & & 0 \\
\hline
\end{tabular}




\begin{tabular}{|c|c|c|c|c|c|}
\hline 产 & 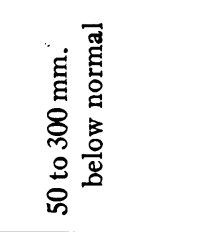 & $\begin{array}{l}\text { 氶 } \\
\text { 台 }\end{array}$ & & 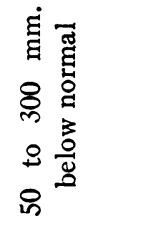 & 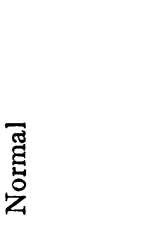 \\
\hline 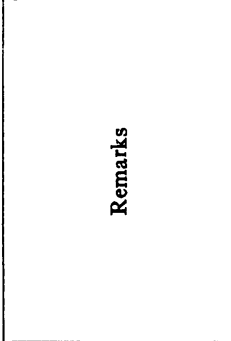 & 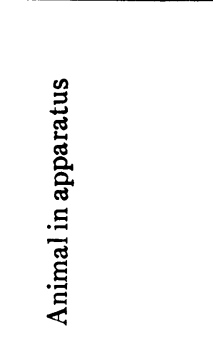 & 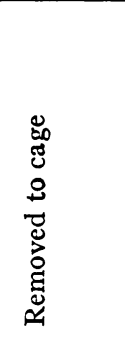 & 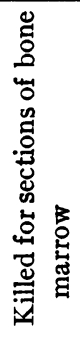 & 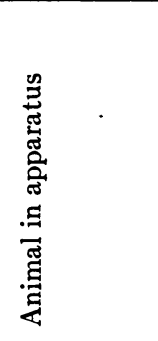 & 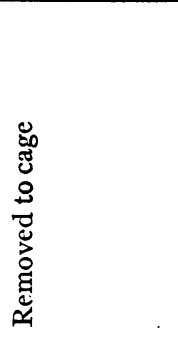 \\
\hline 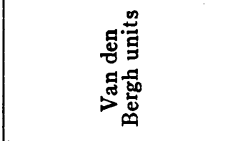 & 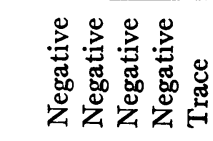 & $\stackrel{-1 N}{=1 N}$ & & 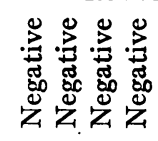 & 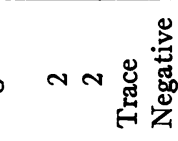 \\
\hline 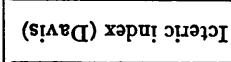 & mamo & $\infty \infty$ & & $\neg-\neg m$ & a a r \\
\hline 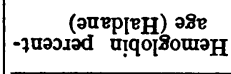 & ஃ゚ ざさ ざ & ㅡㅋ & & ส읓 สำ & ஃ สัสส \\
\hline 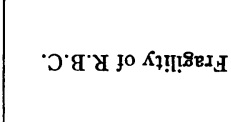 & 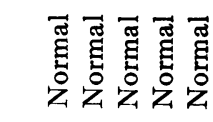 & 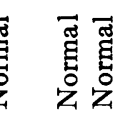 & & 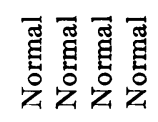 & 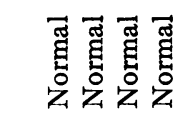 \\
\hline 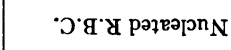 & 00000 & 00 & & 0000 & 0000 \\
\hline 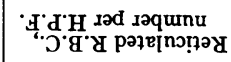 & Ont in t & $m N$ & & $O M$ in & Noo 0 \\
\hline słunos $\cdot \partial \cdot g \cdot y$ & 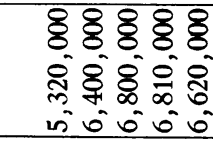 & 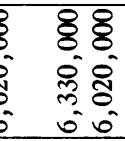 & & 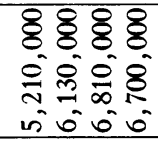 & 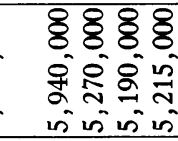 \\
\hline 74:? & 害 융 & & & $\stackrel{n}{\stackrel{0}{N}}$ & \\
\hline 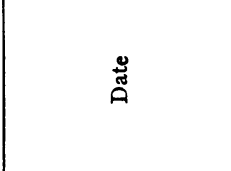 & 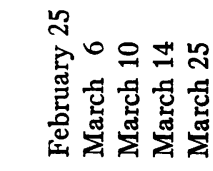 & 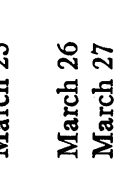 & & 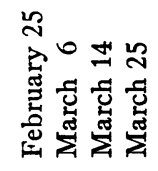 & 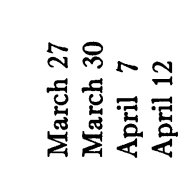 \\
\hline $28 \mathrm{~V}$ & 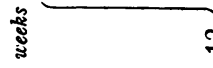 & $\cong$ & & & $\simeq$ \\
\hline 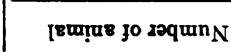 & & n & & & $\infty$ \\
\hline
\end{tabular}




\begin{tabular}{|c|c|c|c|c|c|}
\hline 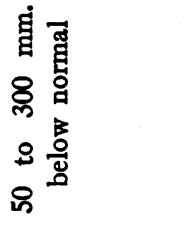 & 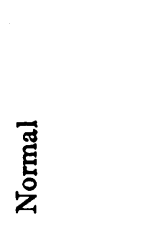 & $\begin{array}{l}\text { 氶 } \\
\text { 乙 }\end{array}$ & 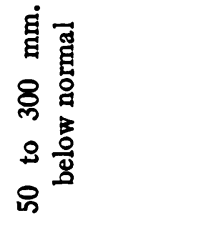 & & 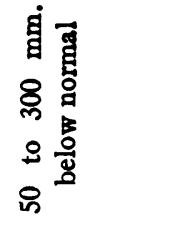 \\
\hline 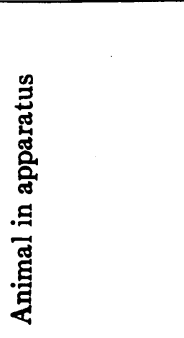 & 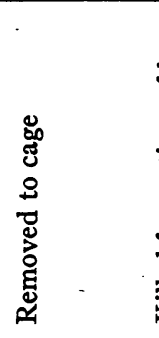 & 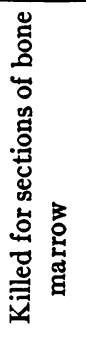 & 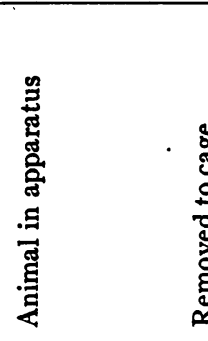 & 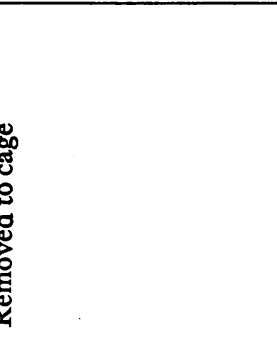 & 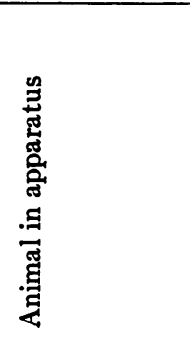 \\
\hline 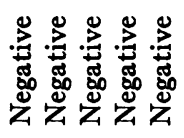 & $\neg \stackrel{-1 N}{\rightarrow}$ & & 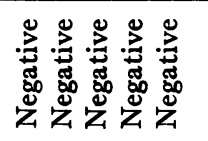 & 曾 & 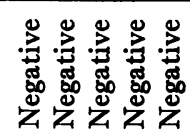 \\
\hline NNNNN & nnt & & ルールーナ & $m n-0 m-7$ & NNNNN \\
\hline ゙윽웡워 & 忍 & & \&읔 & $\stackrel{\infty}{=}$ & ゙す잌의 \\
\hline 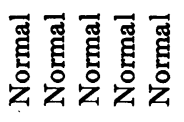 & 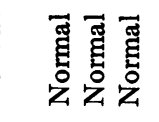 & & 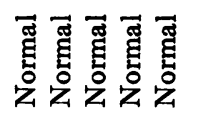 & 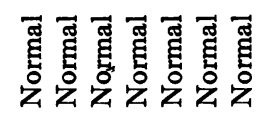 & 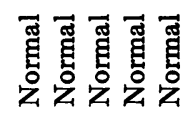 \\
\hline 00000 & 000 & & 00000 & 0000000 & 00000 \\
\hline $0 \pi+00$ & Hen & & $0 \mathrm{~m}$ in 0 & intmo000 & Ominno \\
\hline 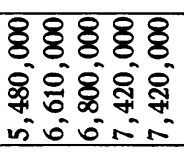 & 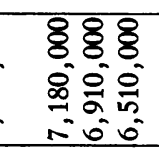 & & 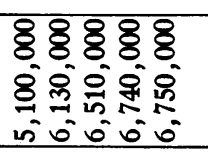 & 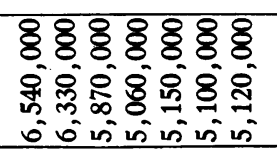 & 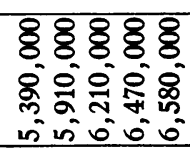 \\
\hline$\stackrel{\sim}{8}$ & & & 芩 & & ஷ্ণ \\
\hline 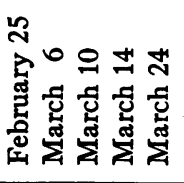 & 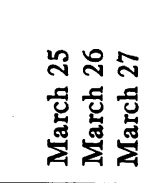 & & 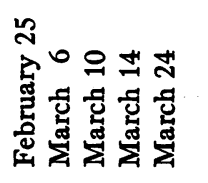 & 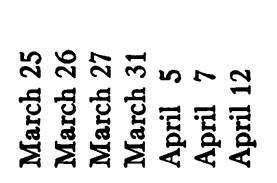 & 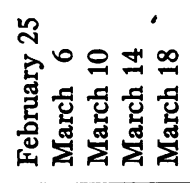 \\
\hline \multicolumn{3}{|c|}{ 웅 } & \multicolumn{2}{|r|}{$\equiv$} & $\approx$ \\
\hline & \multicolumn{2}{|r|}{ 으 } & $\exists$ \\
\hline
\end{tabular}




\begin{tabular}{|c|c|c|c|c|}
\hline 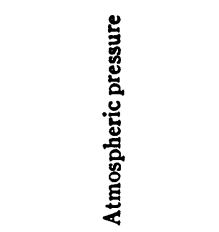 & \multicolumn{2}{|c|}{ 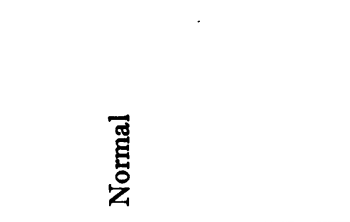 } & $\begin{array}{l}\text { 范 } \\
\text { Z } \\
\end{array}$ & 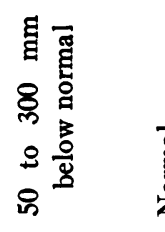 \\
\hline 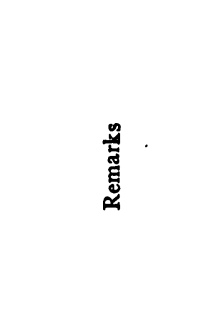 & \multicolumn{2}{|c|}{$\mathscr{q}$} & 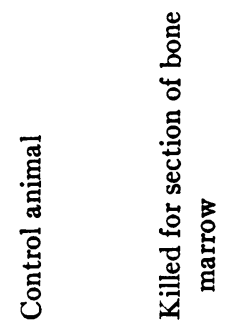 & 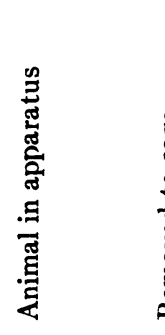 \\
\hline 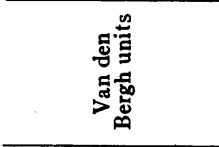 & 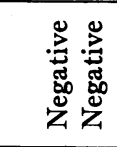 & 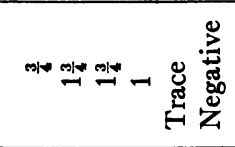 & 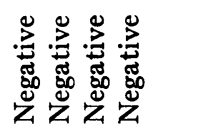 & 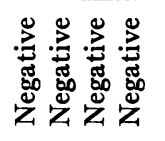 \\
\hline 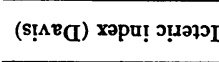 & $N N$ & $0 \infty \infty$ nn & $\neg-\neg-$ & NNNM \\
\hline 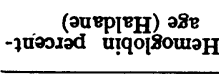 & $\stackrel{ }{=}$ & 总ずよホずず & む゙ず๙゙す & 윯 \\
\hline 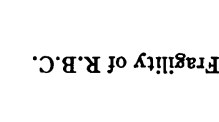 & 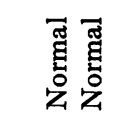 & 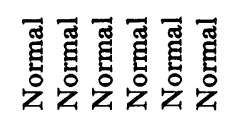 & 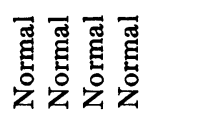 & 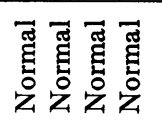 \\
\hline 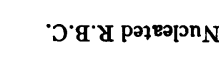 & 00 & 000000 & 0000 & 0000 \\
\hline 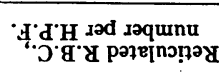 & in in & mnNo0 & 0000 & O $\mathrm{m}$ in in \\
\hline şunos $\cdot \partial \cdot g \cdot y$ & $\begin{array}{ll}8 & 8 \\
8 & 8 \\
8 & 0 \\
0 & 0 \\
0 & 0 \\
0 & 0\end{array}$ & 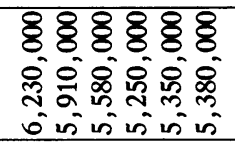 & 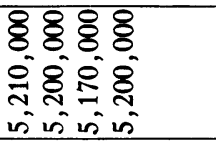 & 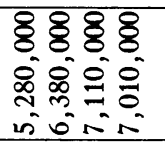 \\
\hline 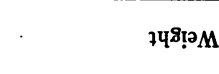 & $\stackrel{8}{8}$ & & $\stackrel{ }{*}$ & $\stackrel{\infty}{\approx}$ \\
\hline 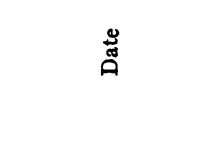 & 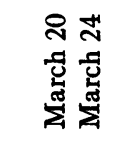 & 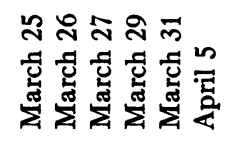 & 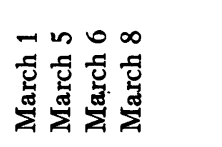 & 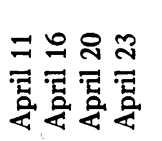 \\
\hline $28 \mathrm{~V}$ & है & $\simeq$ & $\simeq$ & $a$ \\
\hline [ewịu fo saqunnN & & $=$ & $\simeq$ & $\cong$ \\
\hline
\end{tabular}




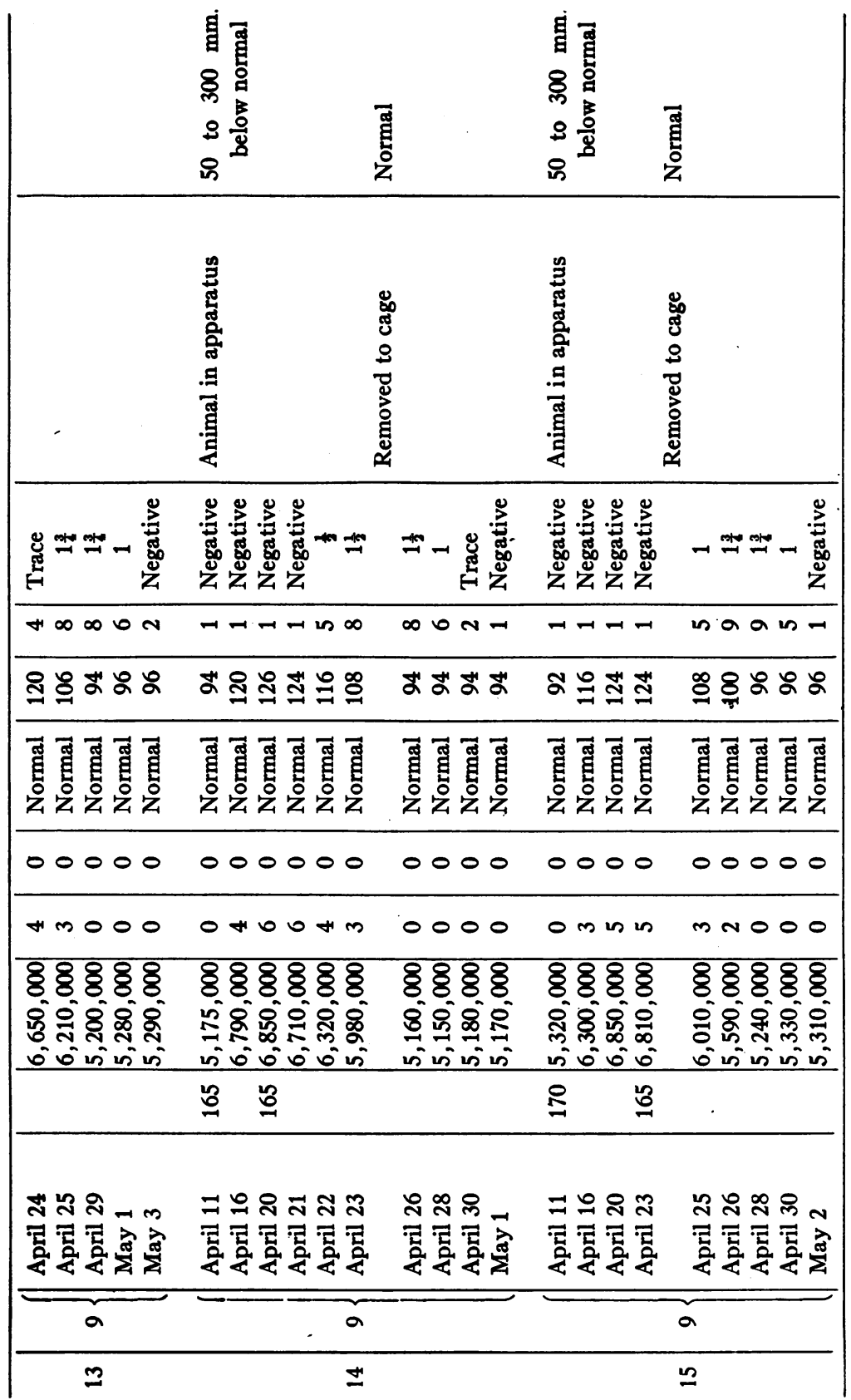




\begin{tabular}{|c|c|c|c|c|}
\hline 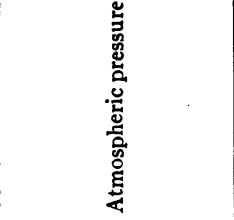 & 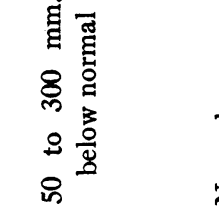 & 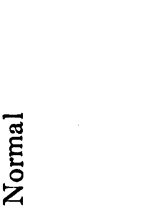 & $\begin{array}{l}\bar{\sigma} \\
\text { हूँ } \\
\text { Zे }\end{array}$ & 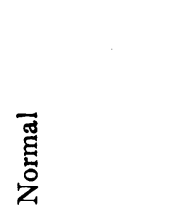 \\
\hline 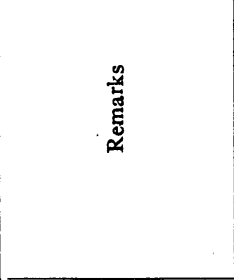 & 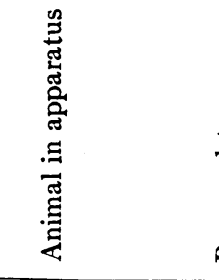 & 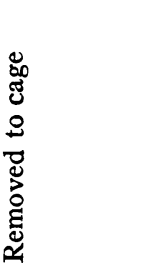 & 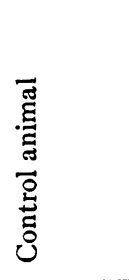 & 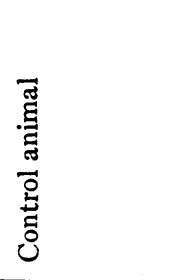 \\
\hline 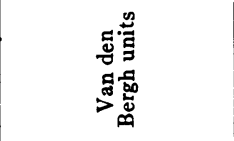 & 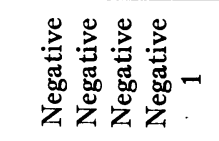 & 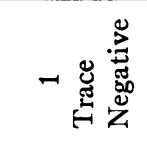 & 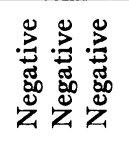 & 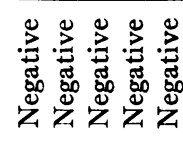 \\
\hline 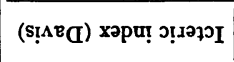 & $\sim \sim \sim m \infty$ & $\infty n \sim$ & $\neg--$ & $\operatorname{NNNN}$ \\
\hline 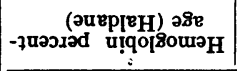 & 영윽웜음 & \& \& \& & สสส & 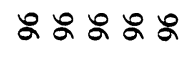 \\
\hline 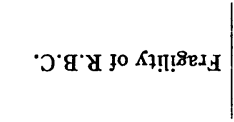 & 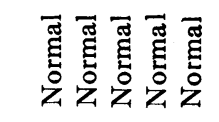 & 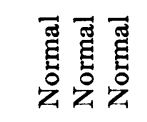 & 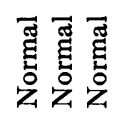 & 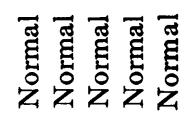 \\
\hline 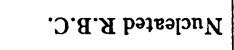 & 00000 & 000 & 000 & 00000 \\
\hline 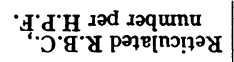 & On m n t n & 000 & 000 & 00000 \\
\hline słunos $\cdot \partial \cdot g \cdot y$ & 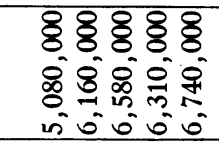 & $\begin{array}{l}8888 \\
88 \\
888 \\
080 \\
\text { nin in }\end{array}$ & 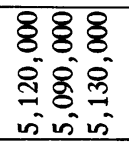 & 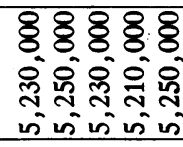 \\
\hline $749 \div 2 M$ & $\stackrel{0}{5} \stackrel{\infty}{=}$ & & $\stackrel{\infty}{\infty}$ & $\stackrel{8}{\sim} \quad \stackrel{2}{-}$ \\
\hline 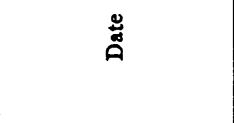 & 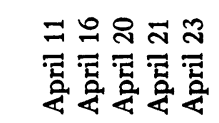 & 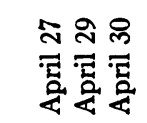 & 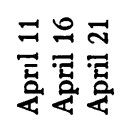 & 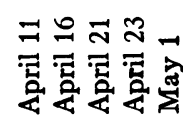 \\
\hline $28 \mathrm{~V}$ & $a$ & & $a$ & $a$ \\
\hline [eurute jo səquin $N$ & 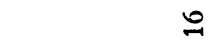 & & $\approx$ & $\stackrel{\infty}{\sim}$ \\
\hline
\end{tabular}




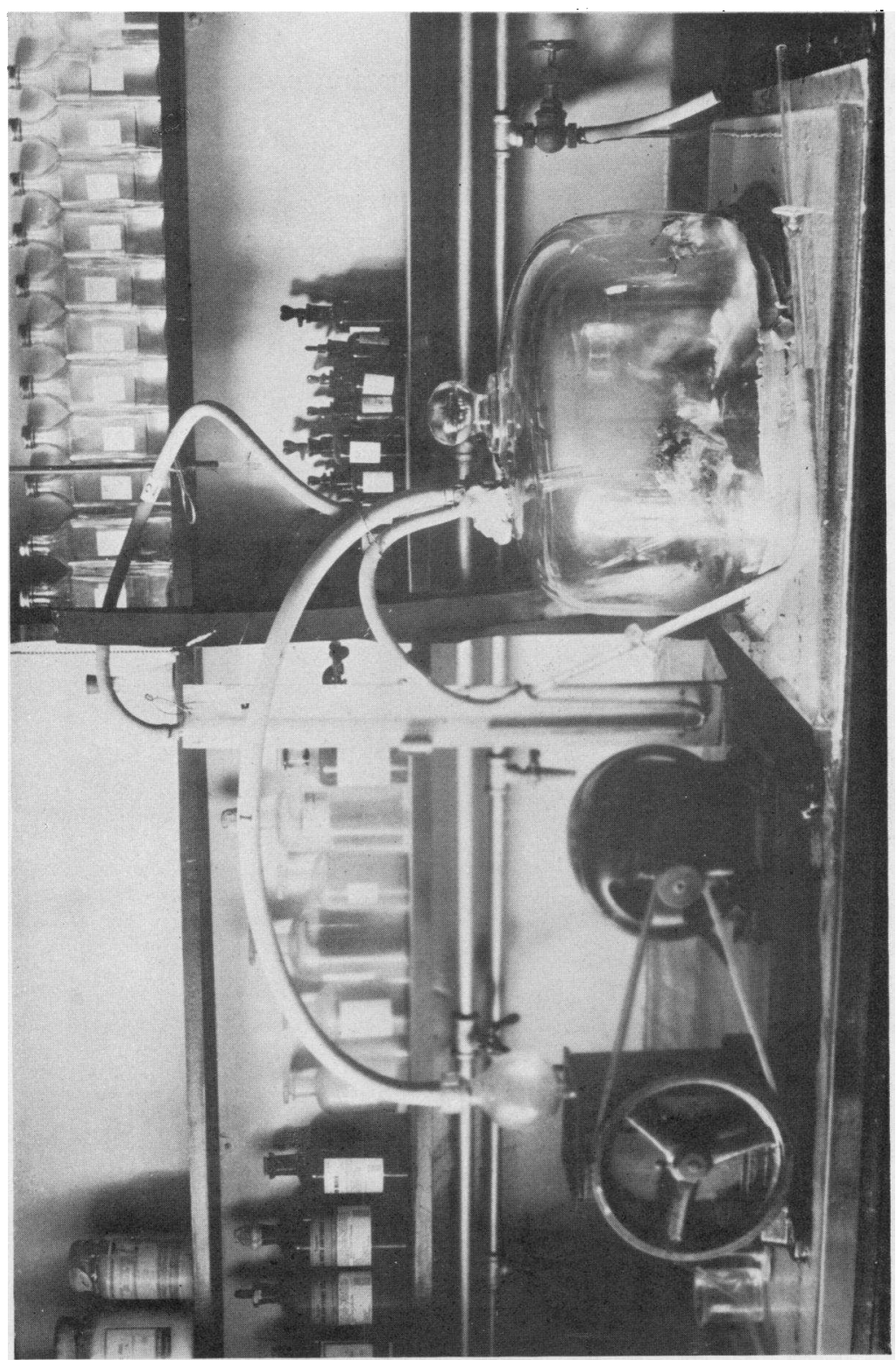

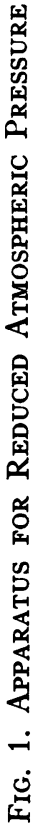


existed, leading to destruction of the excess of red cells and thus to icterus neonatorum.

We felt therefore that if these theories are correct it should be possible not only to produce a polycythaemia by keeping animals under reduced atmospheric pressure but also to produce some degree of jaundice in these animals shortly after the pressure is restored to normal.

Accordingly we have attempted to produce a polycythaemia in

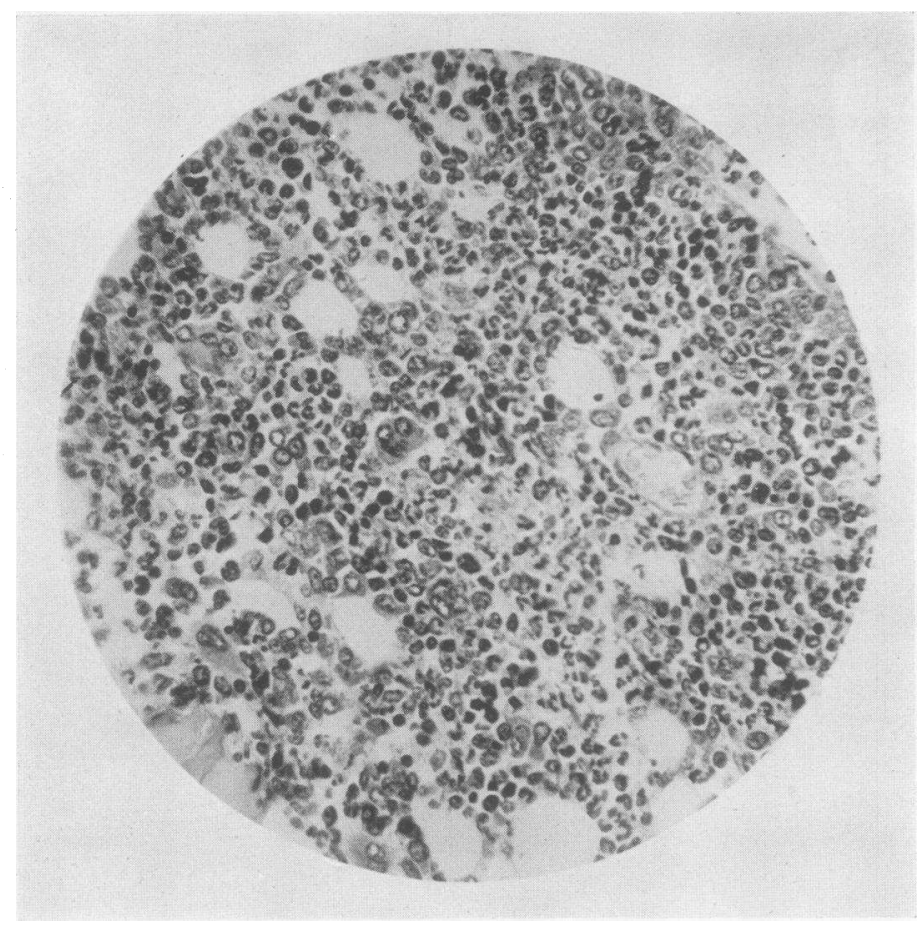

Fig. 2. Bone Marrow of a Normal Guinea Pig

guinea pigs by keeping them under reduced atmospheric pressure by means of a specially devised apparatus (see fig. 1). Tube 1 connects a glass jar, which is fixed air tight to a glass plate, with a vacuum pump. Tube 2 connects the jar with a manometer. Tube 3 allows air to enter, the amount of which could be regulated by a stop cock.

Eighteen young guinea pigs were used for this experiment, thirteen of them were kept from 2 to 4 weeks under reduced atmospheric pres- 
sure while the other five were kept as controls. One animal (no. 1) died at the end of one week in the apparatus, but had already begun to show an increase in the number of red blood cells. Sections of the bone marrow of this animal were made and showed increased activity. A sixth control animal was killed at the beginning of the experiment for bone marrow studies. The pressure in the apparatus was gradu-

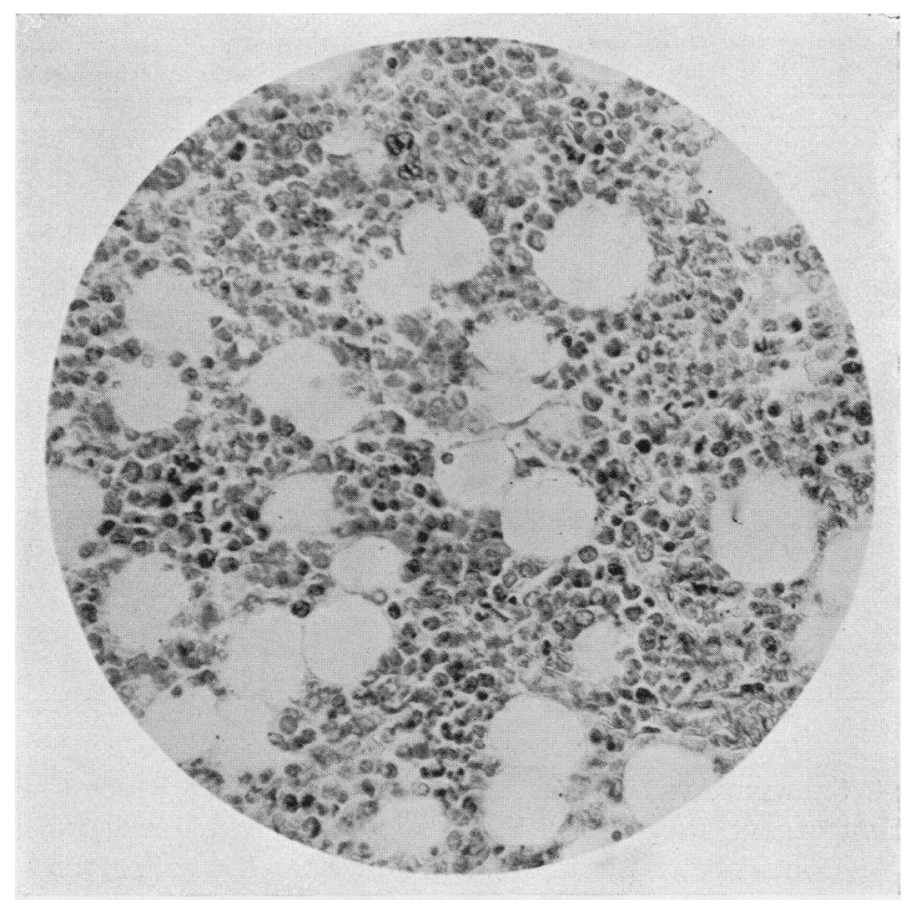

Fig. 3. Bone Marrow of a Guinea Pig after 4 Weeks Exposure to Reduced Atmospheric Pressure

ally reduced to $300 \mathrm{~mm}$. below normal atmospheric pressure and then kept constant at this level, which corresponds to an altitude of about 18,000 feet. Food was introduced daily through the top opening; for that reason and on account of the blood studies the pressure had to be increased every day for a few minutes. A total of about 200 minutes of normal atmospheric pressure was necessary during the 
whole experiment. The blood of the animals was examined every 48 hours. The examinations were made of the number of red blood corpuscles, the percentage of haemoglobin, the number of reticulated and nucleated red blood cells, the fragility of the red blood cells, the icteric index, and the van den Bergh reaction. The blood for the examination was taken by puncture of the ear, except for the van den Bergh reaction, for which $0.5 \mathrm{cc}$. was taken by heart puncture.

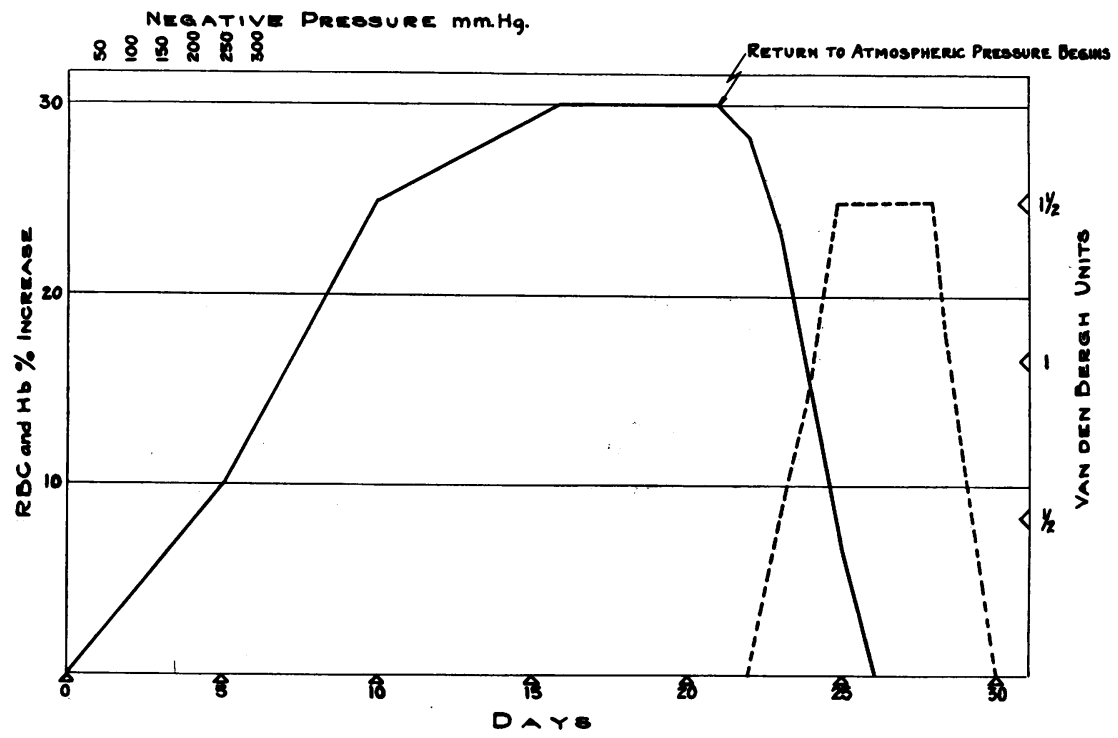

Fig. 4. Showing Average Percentile Change of Red Blood Cei.ls and Hemoglobin, INCREASE in Units of the van DEN BERGH AND the Pressure at Which the Animals were Kept, Plotted Against Days of the Experiments

\section{Red blood cell counts and haemoglobin percentage}

The number of the red blood cells and the percentage of haemoglobin increased rapidly, reaching an average maximum increase of about 30 per cent within 10 days. Five to eight days after the animals were removed to normal atmospheric pressure again, the number of red blood cells and the percentage of haemoglobin returned to their original figures. The chart (fig. 4) shows the average percentile rise of the red blood cells and the haemoglobin, which always ran parallel. 


\section{Reticulated red blood cells}

Reticulated red blood cells were not present in the blood of the animals at normal atmospheric pressure. Three to four days after exposure of the animals to reduced atmospheric pressure, reticulated red blood cells began to appear, reaching in one week the maximal number of 5 to 6 per high power field in thick smears. Within one week after the removal of the animals to normal atmospheric pressure the reticulated red blood cells disappeared again.

Nucleated red blood cells and fragility of the red blood cells

Nucleated red blood cells were at no time present. Fragility of the red blood cells did not show any change during the experiment.

\section{The icteric index and the van den Bergh reaction}

Normal guinea pigs showed an icteric index of from 1 to 3, while the van den Bergh reaction was always negative. The icteric index and the van den Bergh reaction remained unchanged as long as the animals were in the chamber under reduced atmospheric pressure. About 48 hours after the animals were removed from the apparatus to normal atmospheric pressure, the icteric index began to rise and the indirect van den Bergh reaction became positive, both reaching their maximum within 24 hours, an icteric index of from 7 to 9 , and an indirect van den Bergh reaction of from 1 to 2 units. Both icteric index and the van den Bergh reaction remained high for about 8 days and then came gradually down to normal, reaching normal values in 3 to 5 days after the red blood cell count had returned to normal. The table gives a synopsis of the events during the whole experiment.

\section{THE BONE MARROW}

The sections made from the bone marrow of the confined animals showed greater activity than that of the controls (figs. 2 and 3).

\section{SUMMARY OF FINDINGS}

1. Guinea pigs were kept under reduced atmospheric pressure. A polycythaemia developed rapidly, reaching its maximum in about 10 days. The average increase of the red blood cells as well as the haemoglobin was about 30 per cent above the original values. Return to 
normal took place in about 5 to 8 days after the pressure was increased to normal.

2. There was with the polycythaemia a marked increase in reticulated red blood cells, 5 to 6 per high power field. When the pressure was increased to normal the reticulated cells disappeared in about one week.

3. There was an increase in the icteric index and an appearance of positive indirect van den Bergh reactions after the animals were removed from the low atmospheric pressure chamber. The icteric index ranged from 7 to 9 (normal 1 to 3 ) and the van den Bergh reaction from 1 to 2 units (normal 0 ).

\section{COMMENT}

Our previous work demonstrated that the infant develops icterus neonatorum as a result of haemolysis of red blood cells in the course of a period of postnatal re-adjustment, during which the polycythaemia disappears.

The above experiments were designed to produce a polycythaemia in animals through a prolonged diminution of their oxygen supply, and to observe whether icterus occurred during their period of readjustment from polycythaemia to the normal red cell count.

Polycythaemia was successfully produced. As in new born infants, the animals released from the environment of lowered atmospheric pressure, very soon showed an increasing icteric index and a positive indirect van den Bergh reaction together with a reduction of the polycythaemia and the disappearance of the reticulated cells.

Thus an icterus, similar to icterus neonatorum, was produced in animals.

The period of re-adjustment was of somewhat shorter duration than in the human foetus. No nucleated red blood cells were found in these animals and the fragility of their red cells showed no changes.

It would therefore appear quite reasonable to conclude that icterus neonatorum is a haemolytic icterus which is the result of a postnatal re-adjustment from a condition of oxygen unsaturation to a normal oxygen saturation.

\section{BIBLIOGRAPHY}

1. Goldbloom, A., and Gottlieb, R., Am. J. Dis. Child., 1929, xxxviii, 57. Icterus Neonatorum. 